Supporting Information (20 pages)

\title{
Identification of targets of the HIF-1 inhibitor IDF-11774 using alkyne- conjugated photoaffinity probes
}

Hyun Seung Ban ${ }^{1,2 \ddagger}$, Ravi Naik ${ }^{3 \ddagger}$, Hwan Mook Kim ${ }^{4}$, Bo-Kyung Kim ${ }^{5}$, Hongsub Lee ${ }^{6}$, Inhyub Kim ${ }^{5,7}$, Heechul $\mathrm{Ahn}^{3}$, Yerin $\mathrm{Jang}^{3}$, Kyusik Jang ${ }^{3}$, Yumi $\mathrm{Eo}^{3}$, Kyung Bin Song ${ }^{8}$, Kyeong Lee ${ }^{3 *}$, and Misun Won $^{5,7 *}$

${ }^{1}$ Metabolic Regulation Research Center, KRIBB, Daejeon 305-806, Korea

${ }^{2}$ Biomolecular Science, University of Science and Technology, Daejeon 305-350, Korea

${ }^{3}$ College of Pharmacy, Dongguk University-Seoul, Goyang 410-820, Korea

${ }^{4}$ Gachon University, College of Pharmacy, Incheon 406-840, Korea

${ }^{5}$ Personalized Genomic Medicine Research Center, KRIBB, Daejeon 305-806, Korea

${ }^{6}$ ILDONG Pharmaceutical Co. Ltd., Hwaseong, Kyungi-do 445-811, Korea

${ }^{7}$ Functional Genomics, University of Science and Technology, Daejeon 305-350, Korea

${ }^{8}$ Department of Food Science and Technology, Chungnam National University, Daejeon 305-764, Korea

1. Biology

1.1. Table S1

$\begin{array}{ll}\text { 1.2. Figures } \mathrm{S} 1-\mathrm{S} 8 & \mathrm{~S} 3-\mathrm{S} 6\end{array}$

2. Chemistry

2.1. Synthesis of IDF-11774 and Chemical Probes S7-S9

$\begin{array}{lr}\text { 2.2. Figures S9-S18 S10-S19 } & \end{array}$

$\begin{array}{ll}\text { 3. Abbreviations } & \text { S20 }\end{array}$

$\begin{array}{lr}\text { 4. References } & \text { S20 }\end{array}$ 


\section{Biology}

Table S1. Results of mass spectrometric analysis.

\begin{tabular}{|c|c|r|l|}
\hline Residues & $\begin{array}{c}\mathrm{MH}^{+} \\
\text {(Observed) }\end{array}$ & $\begin{array}{c}\text { Mr } \\
\text { (Calculated) }\end{array}$ & \multicolumn{1}{|c|}{ Sequence in heat shock protein 70 } \\
\hline $26-36$ & 614.8223 & 1227.6207 & K.VEIIANDQGNR.T \\
\hline $37-49$ & 744.3594 & 1486.6940 & R.TTPSYVAFTDTER.L \\
\hline $57-71$ & 829.9454 & 1657.8424 & K.NQVALNPQNTVFDAK.R \\
\hline $160-171$ & 599.3567 & 1196.6877 & K.DAGVIAGLNVLR.I \\
\hline $172-187$ & 844.4688 & 1686.8940 & R.IINEPTAAAIAYGLDR.T \\
\hline $221-236$ & 559.2437 & 1674.7234 & K.ATAGDTHLGGEDFDNR.L \\
\hline $349-357$ & 555.3039 & 1108.5665 & K.LLQDFFNGR.D \\
\hline $452-458$ & 401.2212 & 800.4140 & K.DNNLLGR.F \\
\hline $501-507$ & 402.7266 & 803.4389 & K.ITITNDK.G \\
\hline $501-509$ & 509.2854 & 1016.5614 & K.ITITNDKGR.L \\
\hline $510-517$ & 502.2791 & 1002.5345 & R.LSKEEIER.M \\
\hline $518-524$ & 417.6999 & 833.3953 & R.MVQEAEK.Y \\
\hline $536-550$ & 563.6008 & 1687.8239 & R.VSAKNALESYAFNMK.S + Oxidation (M) \\
\hline $540-550$ & 644.3166 & 1286.5965 & K.NALESYAFNMK.S \\
\hline $540-550$ & 652.3134 & 1302.5914 & K.NALESYAFNMK.S + Oxidation (M) \\
\hline $551-559$ & 474.2433 & 946.4607 & K.SAVEDEGLK.G \\
\hline
\end{tabular}


(A)
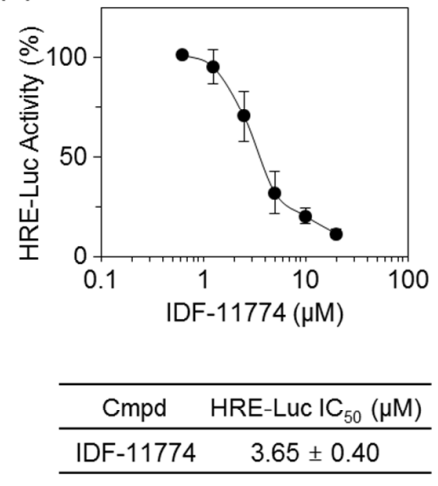

(B)

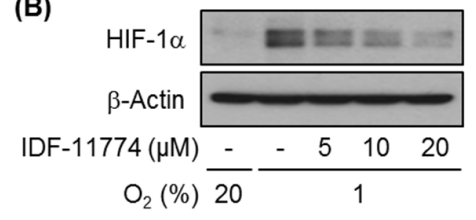

(C)

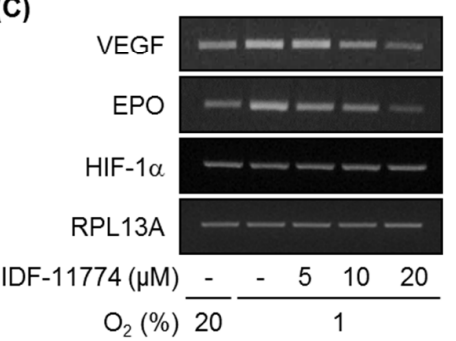

Figure S1. IDF-11774 inhibits HIF-1 $\alpha$ accumulation in HCT116 cells. (A) Effect of IDF-11774 on HRE-luciferase activity. (B) Effect on HIF-1 $\alpha$ accumulation determined by Western blot analysis (C) The effect of IDF-11774 on the mRNA expression of HIF-1 $\alpha$ target genes by RT-PCR.

(A)

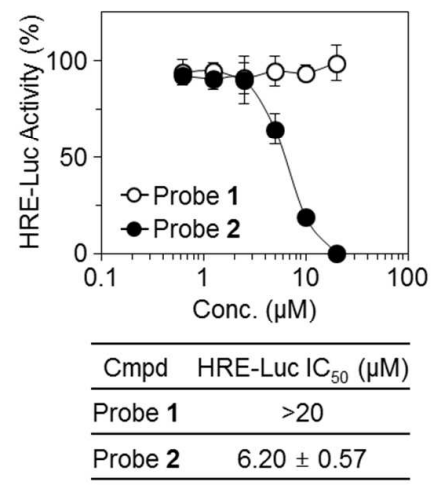

(B)

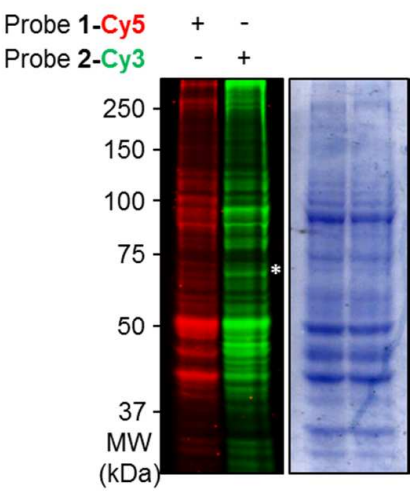

Figure S2. Identification of a target protein of IDF-11774 using chemical probes. (A) HRE-luciferase activity of IDF-11774 probes. (B) Photoaffinity labeling of proteins with probes was followed by click conjugation with a fluorescent dye (Probe 1-Cy5 and probe 2-Cy3). The proteins were separated by SDS-PAGE and visualized by in-gel fluorescence scanning. 
(A) HSP70 NBD (1-388)

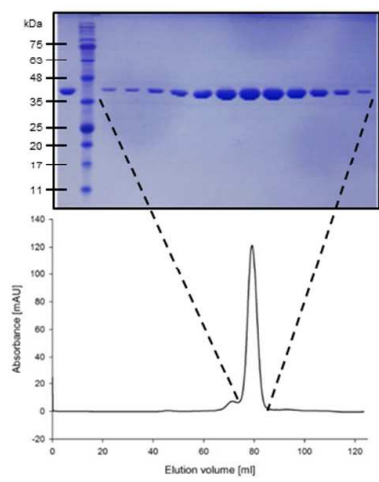

(B) HSP70 SBD (386-613)

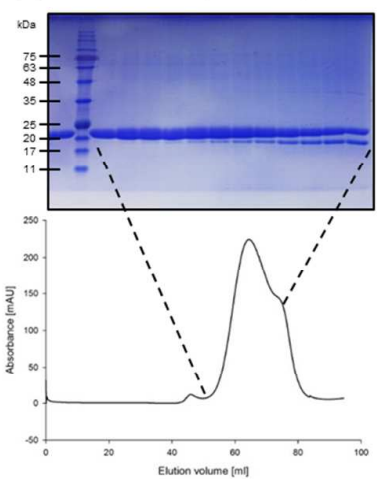

(C) HSP70 FL (1-613)

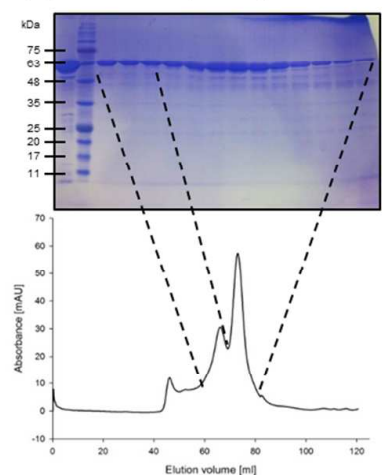

Figure S3. Preparation of recombinant HSP70 proteins. Preparation of recombinant HSP70 proteins. Chromatograms and SDS-PAGE analysis of the eluted fractions from the final size exclusion chromatography step. (A) HSP70 NBD (1-388). (B) HSP70 SBD (386-613). (C) HSP70 FL (1-613).

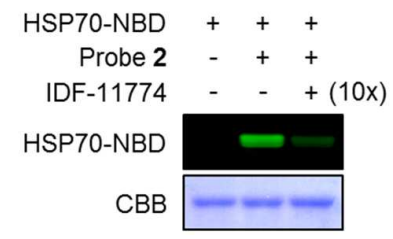

Figure S4. In vitro binding of probe 2 to HSP70-NBD and competition assay with IDF-11774 (100 $\mu \mathrm{M})$.

(A)

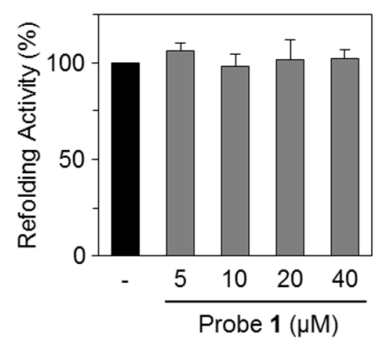

(B)

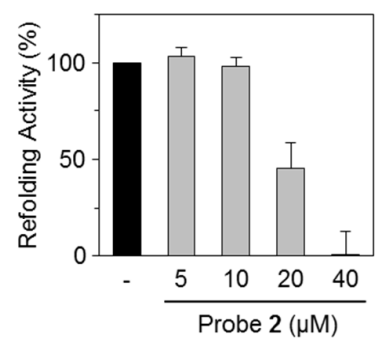

Figure S5. Effects of probes $\mathbf{1}$ and $\mathbf{2}$ on the chaperone activity of HSP70. Guanidine-denatured luciferase (125 ng) was incubated with HSP70 $(0.5 \mu \mathrm{g})$ and HSP40 $(0.3 \mu \mathrm{g})$, and the activity of refolded luciferase was then determined. 
(A)

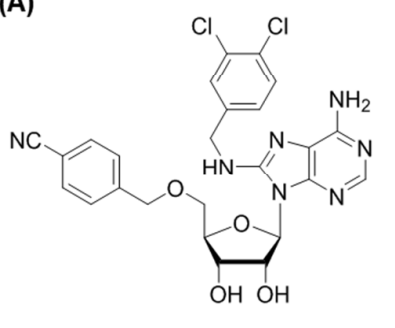

VER-155008
(B)

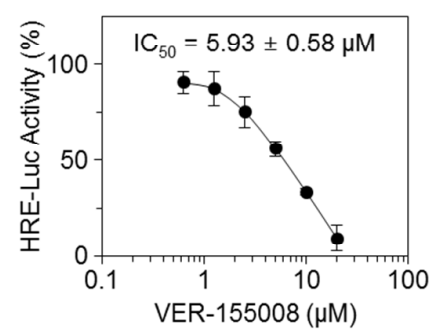

(C)

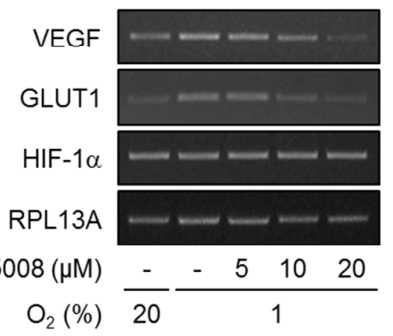

Figure S6. HIF-1 $\alpha$ inhibitory activity of VER-155008. (A) Chemical structure of the HSP70 inhibitor VER-155008. (B) Inhibition of the HRE-Luciferase activity by VER-155008. (C) The effects of VER155008 on the mRNA levels of HIF-1 $\alpha$ target genes by RT-PCR.

(A)

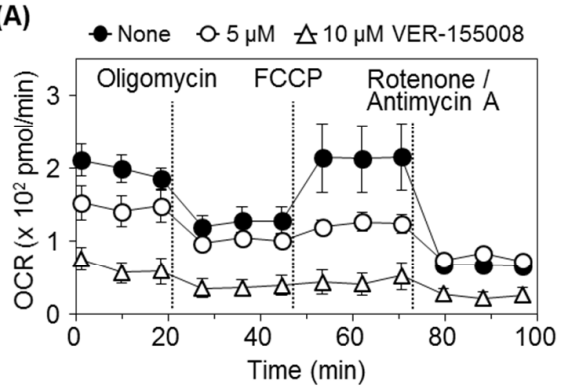

(B)

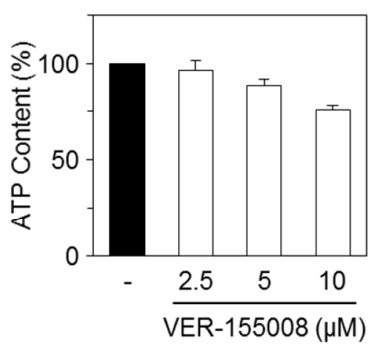

Figure S7. Effect of VER-155008 on mitochondrial metabolism. (A) The effect of VER-155008 on mitochondrial respiration. OCR was measured by adding oligomycin $(1 \mu \mathrm{M})$, FCCP $(0.5 \mu \mathrm{M})$, and rotenone $(1 \mu \mathrm{M}) /$ antimycin A $(1 \mu \mathrm{M})$ to HCT116 cells. (B) Effect of VER-155008 on intracellular ATP content. 
(A)

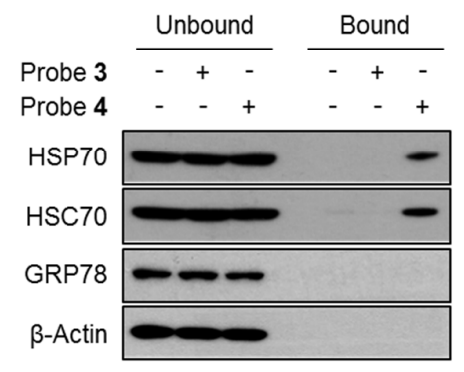

(B)
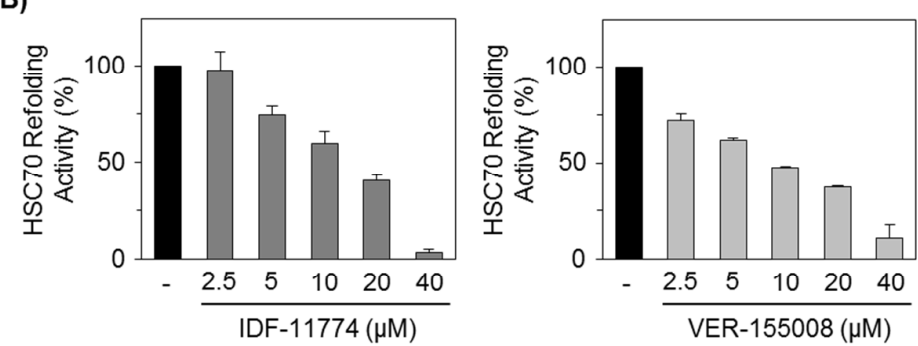

(C)

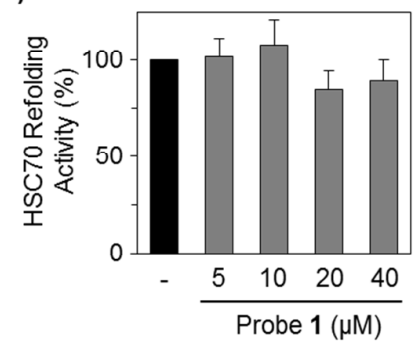

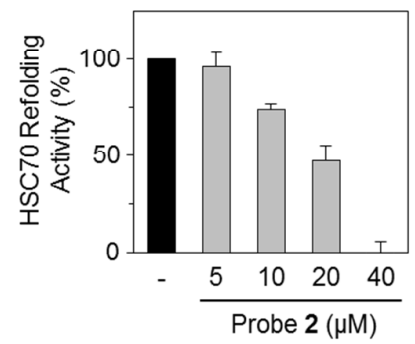

Figure S8. Inhibition of HSC70 by IDF-11774. (A) Binding of IDF-11774 to HSP70 isotype. Pulldown assay using IDF-11774-biotin probe or control probe was carried out and HSP70 isoforms were detected by Western blot analysis. (B) The effects of IDF-11774 and VER-155008 on HSC70 chaperone activity. (C) The effects of chemical probes of IDF-11774 on HSC70 chaperone activity. Guanidine-denatured luciferase (125 ng) was incubated with HSC70 $(0.5 \mu \mathrm{g})$ and HSP40 (0.3 $\mu \mathrm{g})$, and the activity of refolded luciferase was determined. 


\section{Chemistry}

\subsection{Synthesis of IDF-11774 and Chemical Probes}

Scheme S1 Synthesis of IDF-11774. ${ }^{\mathrm{a}}$

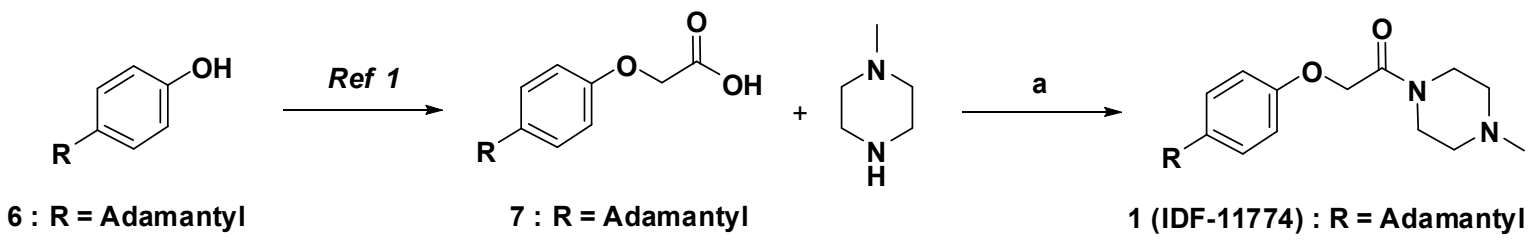

${ }^{a}$ Reagents and Conditions: a) EDC HCl, HOBT, DIPEA, DMF.

Scheme S2 Synthesis of diazirine chemical probes $\mathbf{1}$ and 2. ${ }^{\mathrm{a}}$

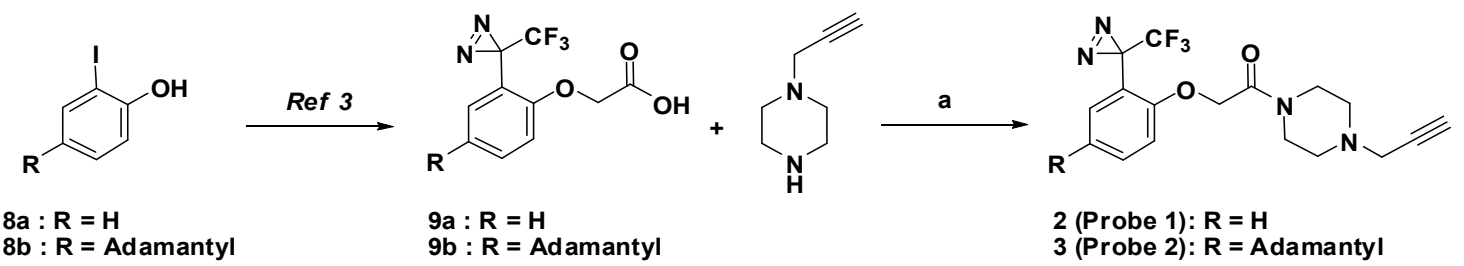

${ }^{a}$ Reagents and Conditions: a) EDC $\mathrm{HCl}$, HOBt, DIPEA, DMF. 
Scheme S3 Synthesis of biotin chemical probes 3 and $4 .^{a}$
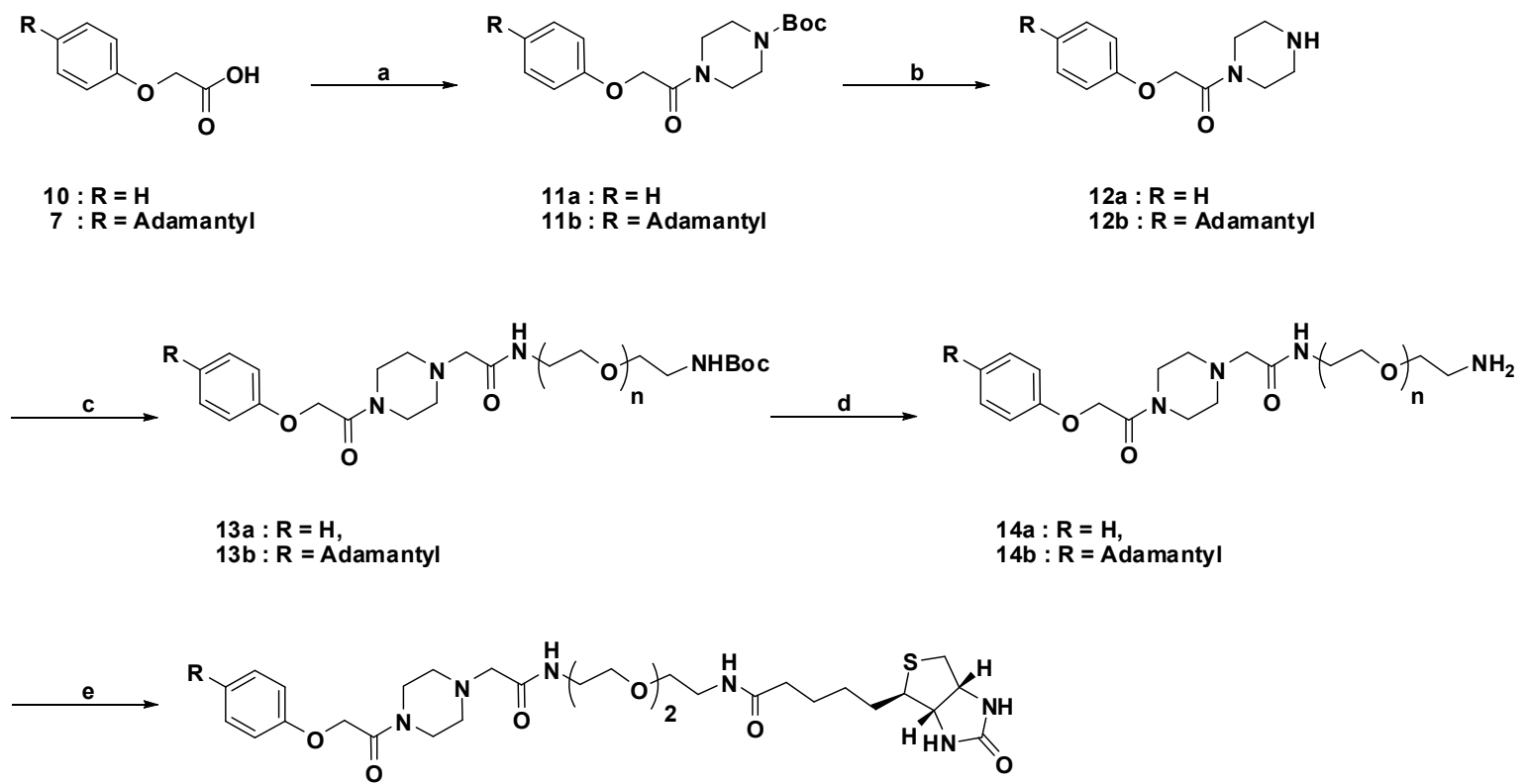

4 (Probe 3): $\mathbf{R}=\mathbf{H}$,

5 (Probe 4) : R = Adamantyl

${ }^{a}$ Reagents and Conditions: a) 1-Boc piperizine, HBTU, DIPEA, DMF; b) TFA, MC; c) $\mathrm{K}_{2} \mathrm{CO}_{3}$, $\mathrm{Cs}_{2} \mathrm{CO}_{3}, \mathrm{KI}$, acetone/DMF, (2-\{2-[2-(2-Chloro-acetylamino)-ethoxy]-ethoxy\}-ethyl)-carbamic acid tert-butyl ester; d) TFA, MC; e) TEA, DMF, (+)-Biotin $N$-hydroxysuccinimide ester.

The compound IDF-11774 and chemical probes 1-4 were synthesized as shown in Schemes S1-S3. 
The compound IDF-11774 was synthesized via acid-amine coupling between 1-methyl piperazine and 4-(1-adamantyl)-phenoxy acetic acid (Scheme S1). ${ }^{1}$

Trifluoromethyl diazirine has several valuable characteristics, including photoactivity at longer wavelengths, good chemical stability, rapid photolysis, and generation of a highly reactive carbene. For the synthesis of the trifluoromethyl diazirine probe $\mathbf{2}$, a key intermediate $\mathbf{9 b}$ was readily acquired by our previously reported procedure. Reaction between $\mathbf{9 b}$ and 1-(prop-2-ynyl)piperazine led to the desired chemical probe 2 . Meanwhile, the negative analogue probe $\mathbf{1}$ was also prepared by the same method via 9a, starting from commercially available 2-iodo anisole $\mathbf{8 a}$ (Scheme S2). ${ }^{2}$

The syntheses of biotin chemical probes $\mathbf{3}$ and $\mathbf{4}$ carrying structural units like biotin reporter groups began with $\mathbf{7}$ or $\mathbf{1 0}$, which could be readily synthesized via a known procedure. ${ }^{1,2}$ Acid-amine coupling followed by Boc deprotection gave 12a and 12b from 11a and 11b, respectively. In brief, 12b was directly coupled with (2-\{2-[2-(2-Chloro-acetylamino)-ethoxy]-ethoxy $\}$-ethyl)-carbamic acid tert-butyl ester under basic conditions to yield an adamantyl substituent carrying linker 13b. This derivative was further subjected for Boc deprotection to afford 14b. Biotinylation of compound 14b with (+)-biotin $N$-hydroxysuccinimide ester resulted in our desired biotin probe 4 . In contrast, its negative analogue biotin probe 3 was prepared in a similar approach starting from 12a (Scheme S3). ${ }^{2}$ 


\subsection{Figures S1-S10}

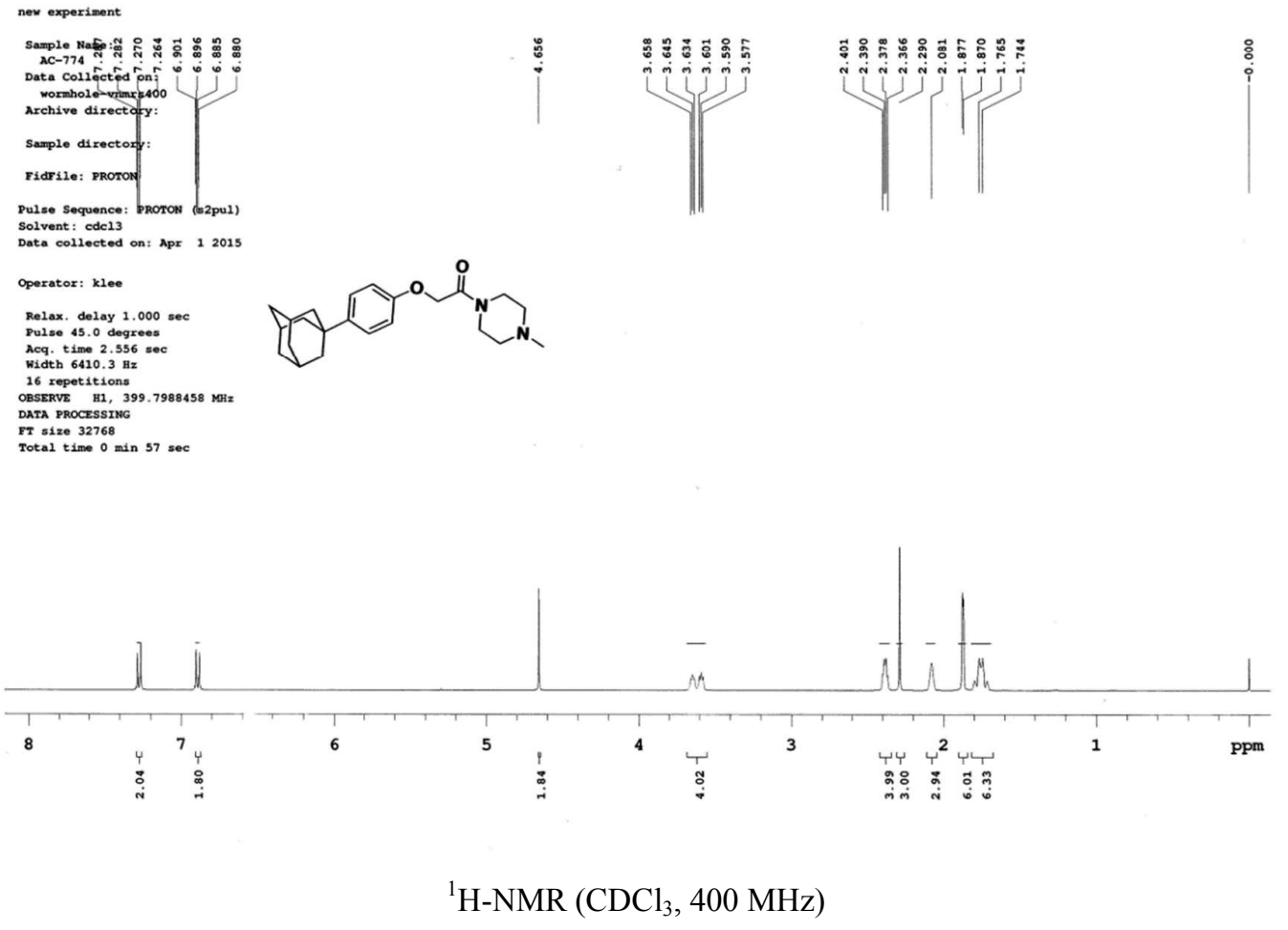

Figure S9. ${ }^{1}$ H-NMR spectrum of 2-(4-adamantan-1-yl-phenoxy)-1-(4-methylpiperazin-1-yl)ethanone (IDF-11774). 


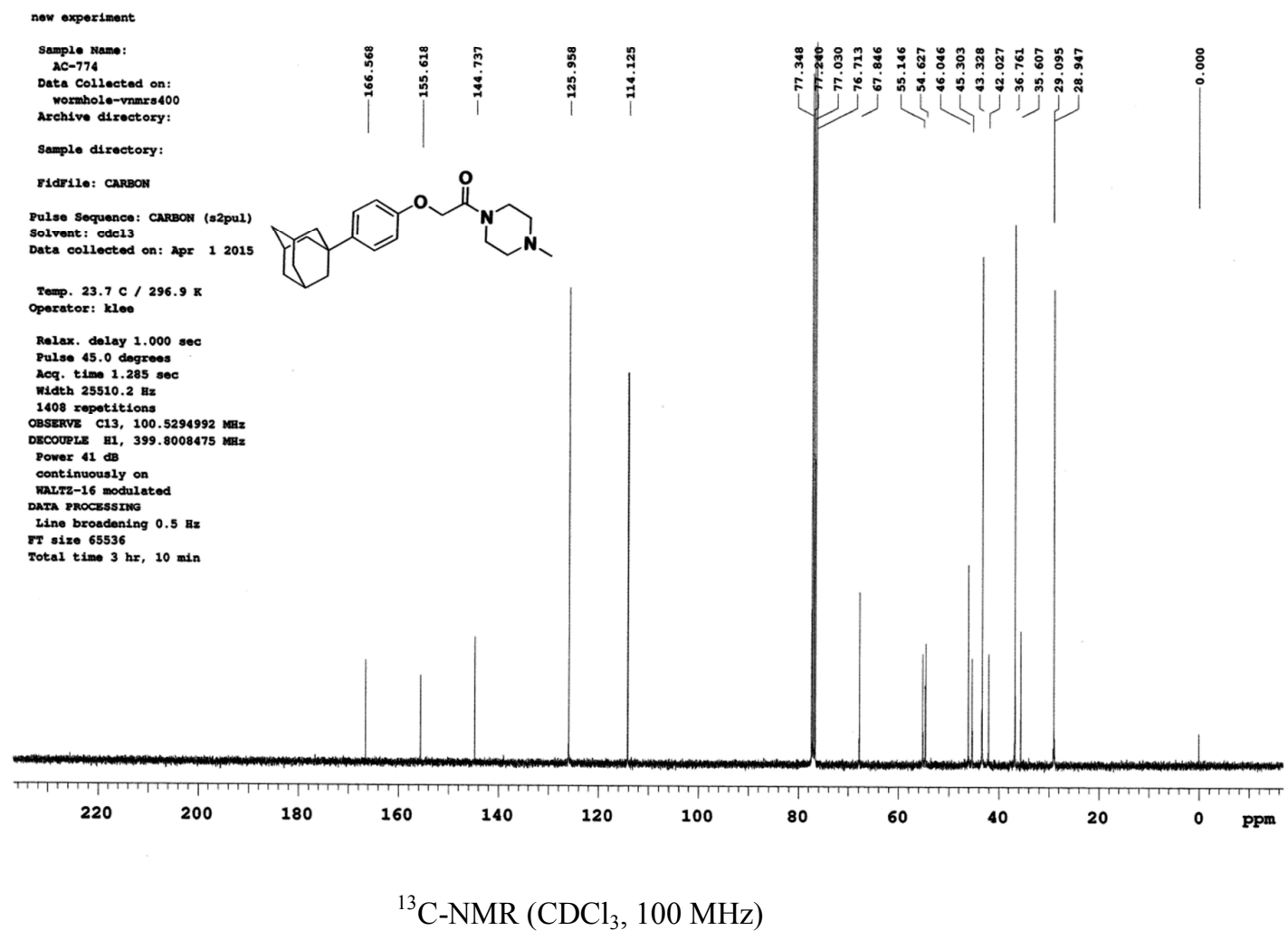

Figure S10. ${ }^{13} \mathrm{C}-\mathrm{NMR}$ spectrum of 2-(4-adamantan-1-yl-phenoxy)-1-(4-methylpiperazin-1-yl)ethanone (IDF-11774). 


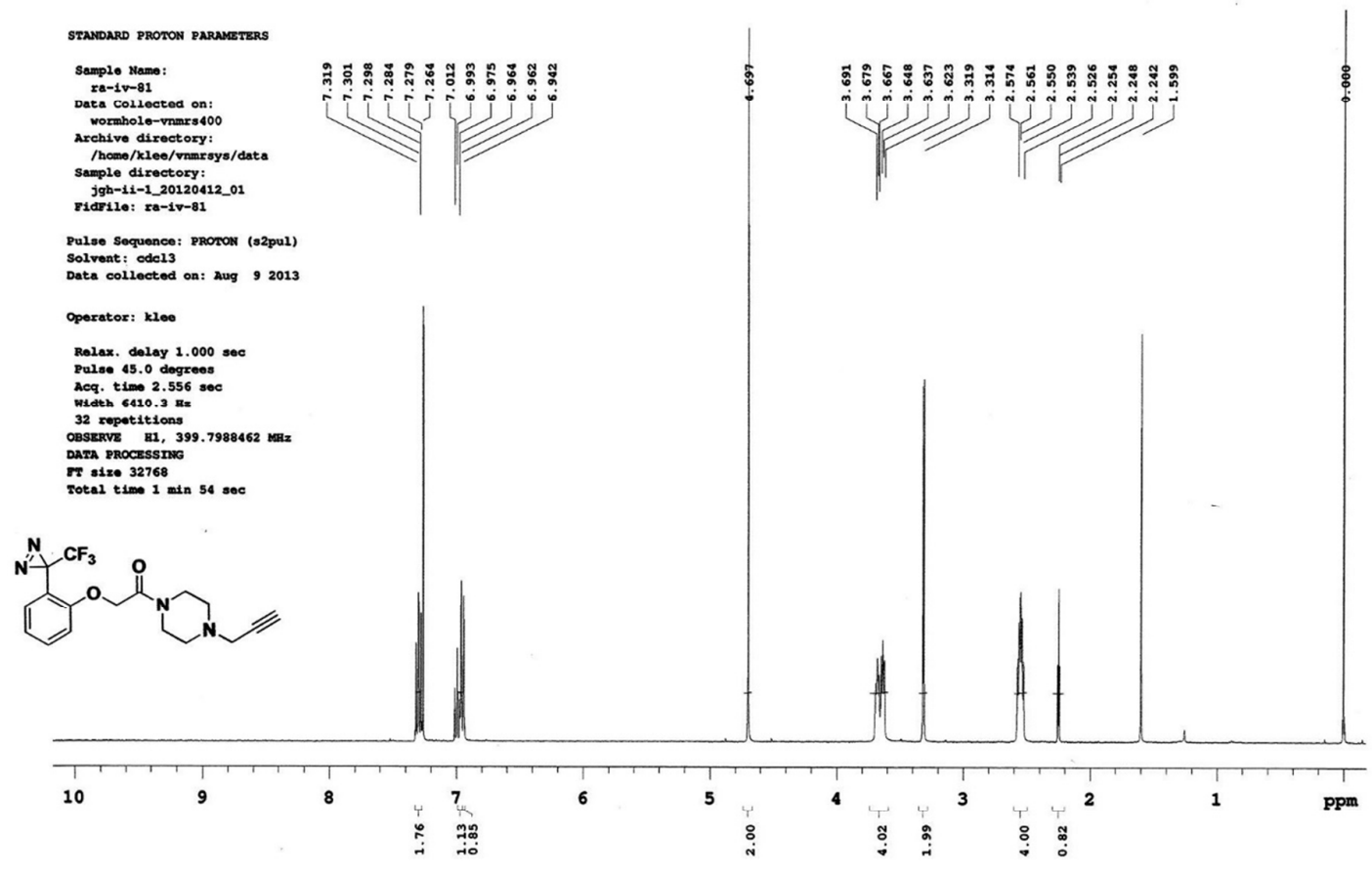

${ }^{1} \mathrm{H}-\mathrm{NMR}\left(\mathrm{CDCl}_{3}, 400 \mathrm{MHz}\right)$

Figure S11. ${ }^{1} \mathrm{H}-\mathrm{NMR}$ spectrum of 1-(4-(prop-2-ynyl)piperazin-1-yl)-2-(2-(3-(trifluoromethyl)-3Hdiazirin-3-yl)phenoxy)ethanone (Probe 1). 


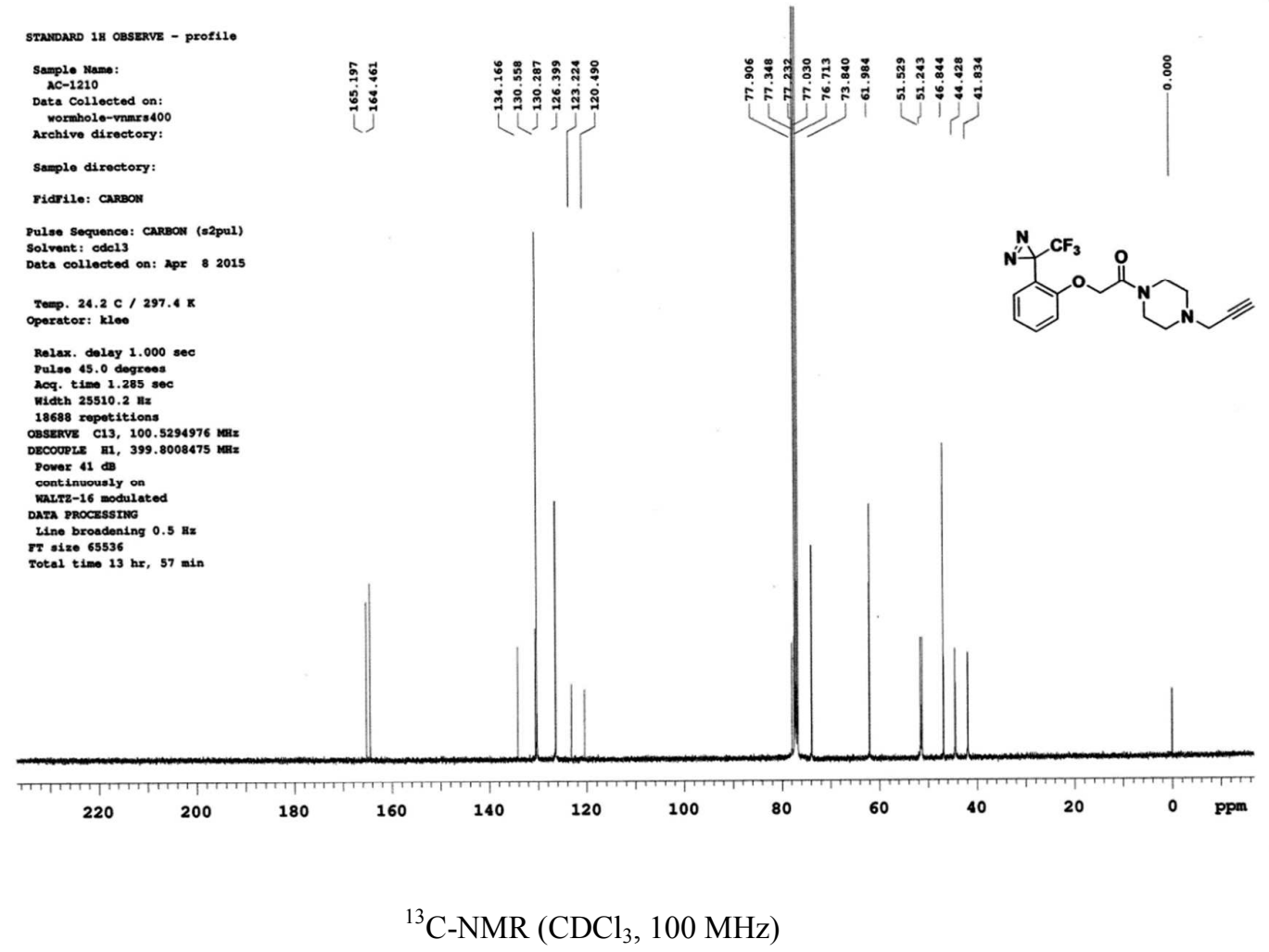

Figure S12. ${ }^{13}$ C-NMR spectrum of 1-(4-(prop-2-ynyl)piperazin-1-yl)-2-(2-(3-(trifluoromethyl)-3Hdiazirin-3-yl)phenoxy)ethanone (Probe 1). 


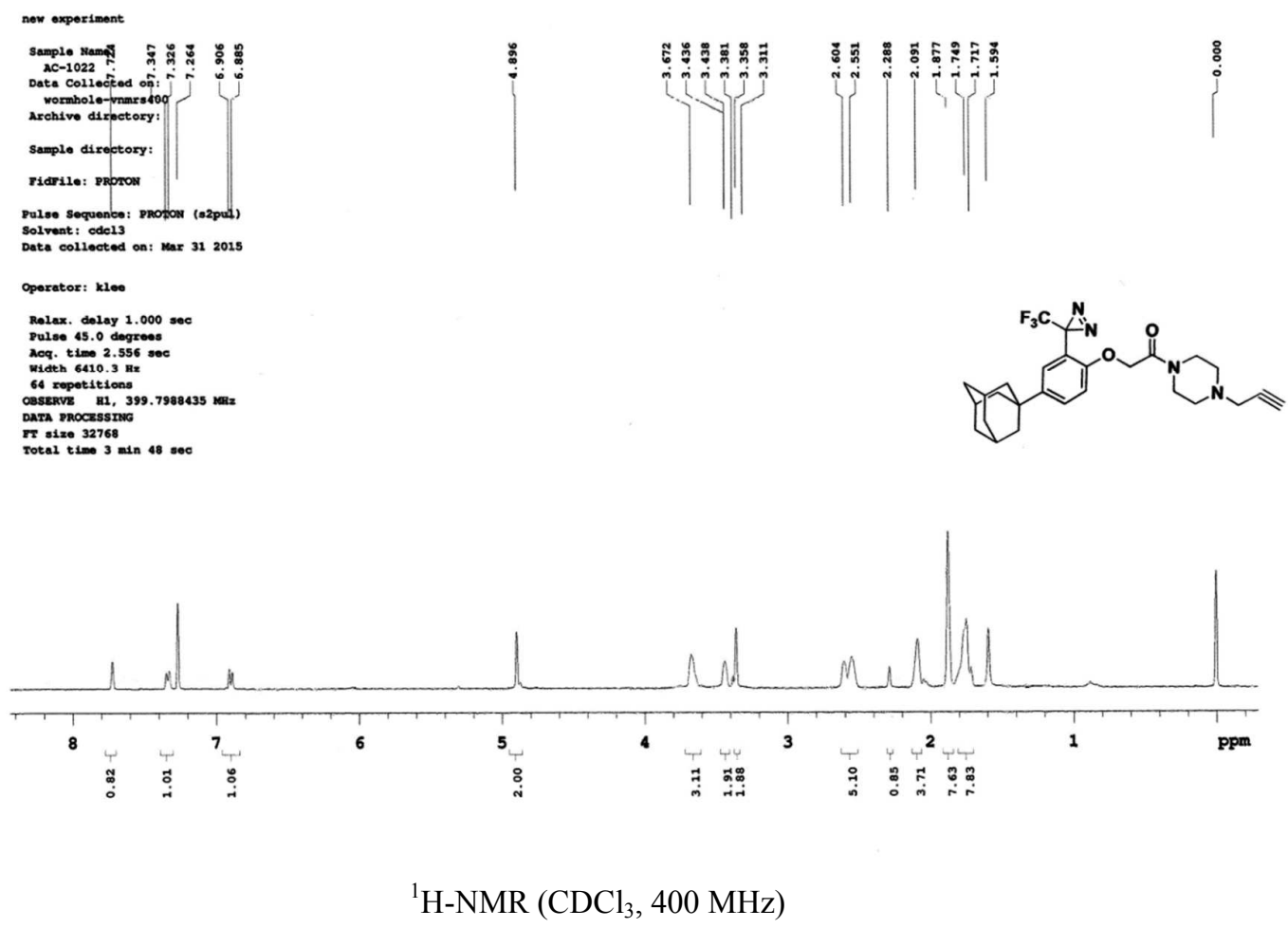

Figure S13. ${ }^{1}$ H-NMR spectrum of 2-(4-adamantan-1-yl-2-(3-(trifluoromethyl)-3H-diazirin-3-yl)phenoxy)-1(4-(prop-2-ynyl)piperazin-1-yl)ethanone (Probe 2). 


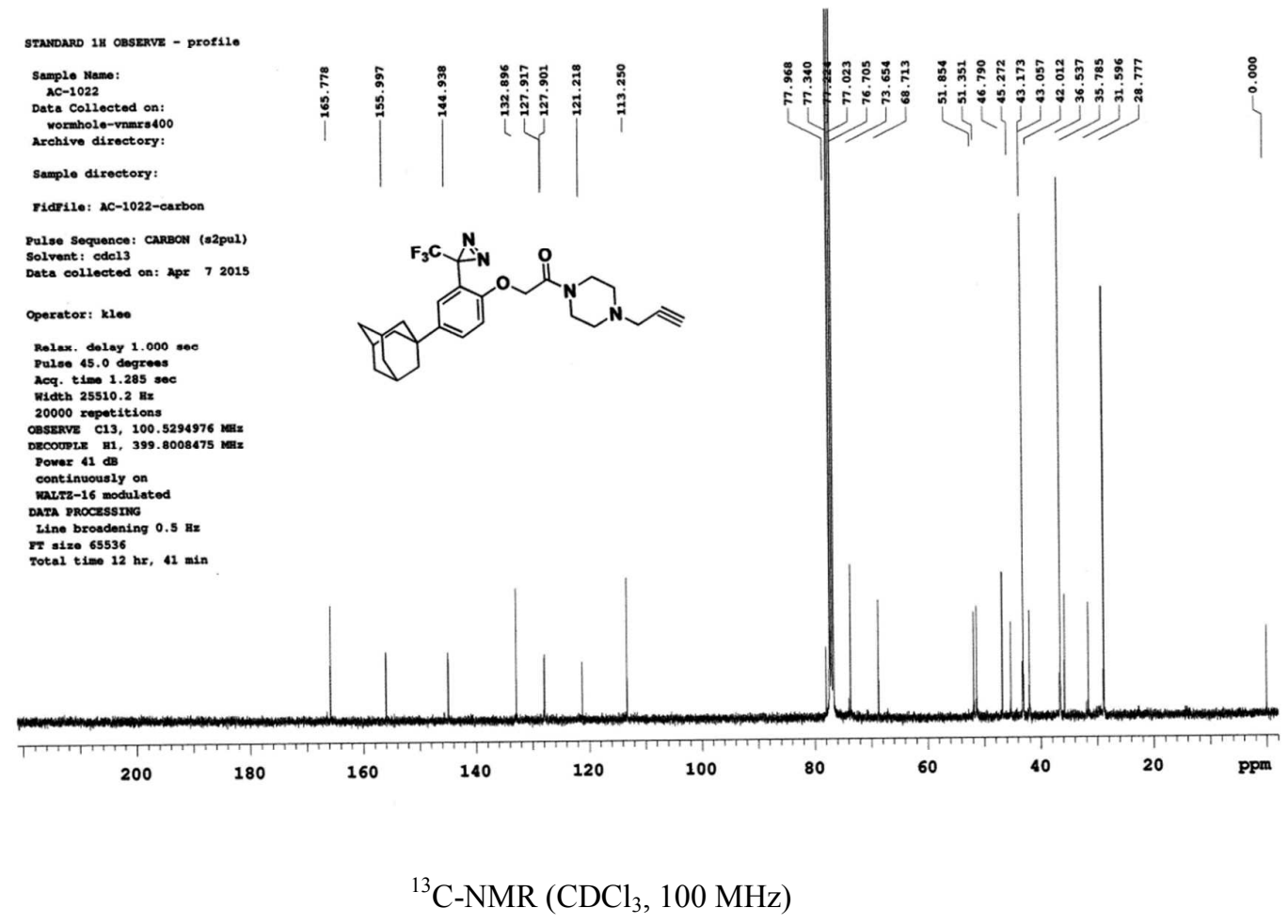

Figure S14. ${ }^{13} \mathrm{C}-\mathrm{NMR}$ spectrum of 2-(4-adamantan-1-yl-2-(3-(trifluoromethyl)-3H-diazirin-3-yl)phenoxy)-1(4-(prop-2-ynyl)piperazin-1-yl)ethanone (Probe 2). 


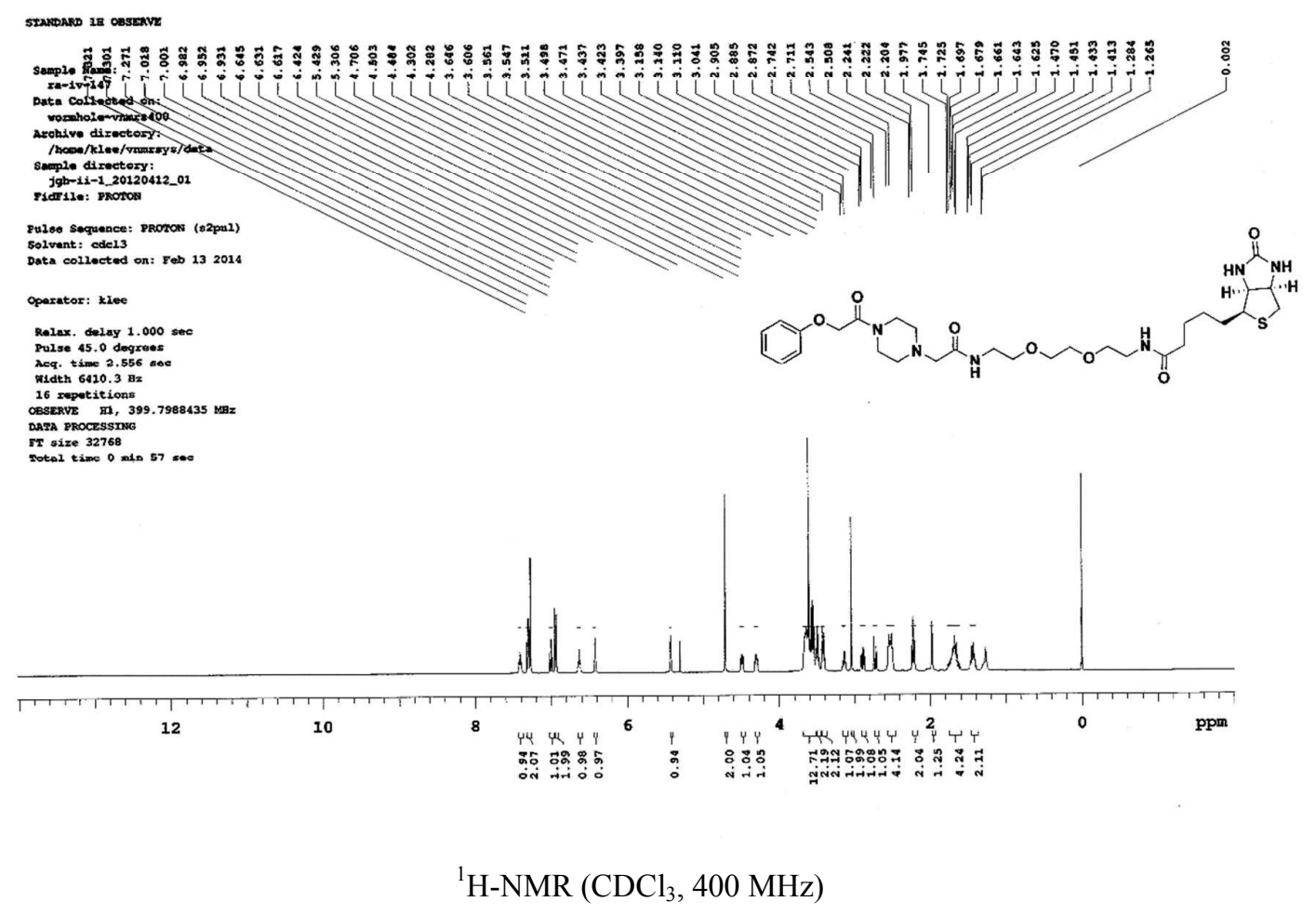

Figure S15. ${ }^{1} \mathrm{H}-\mathrm{NMR}$ spectrum of 5-((3aS,4S,6aR)-2-oxohexahydro-1H-thieno[3,4-d]imidazol-4-yl)- $N$ (2-(2-(2-(2-(4-(2-phenoxyacetyl)piperazin-1-yl)acetamido)ethoxy)ethoxy)ethyl)pentanamide (Probe 3). 


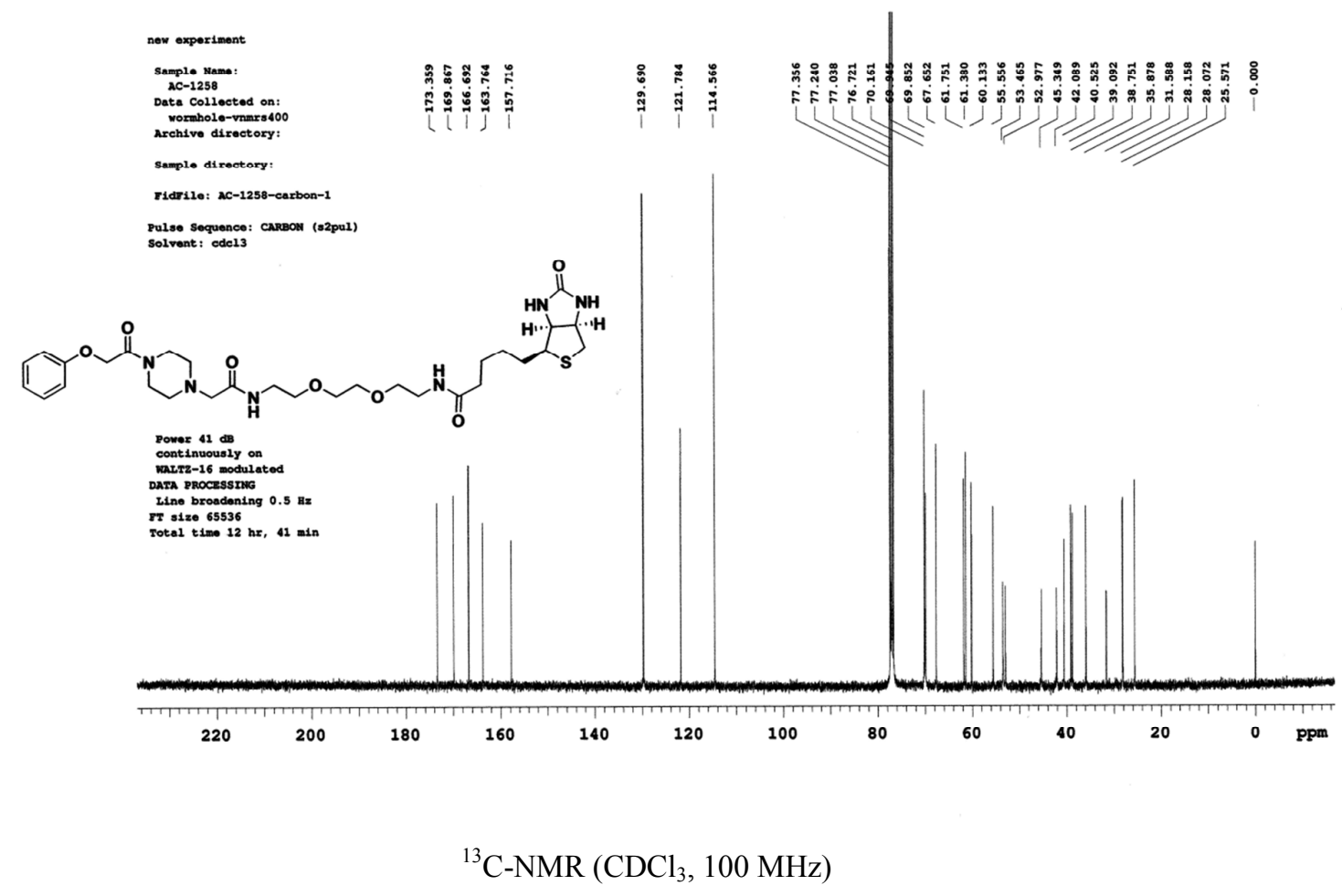

Figure S16. ${ }^{13} \mathrm{C}-\mathrm{NMR}$ spectrum of 5-((3aS,4S,6aR)-2-oxohexahydro-1H-thieno[3,4-d]imidazol-4-yl)$N$-(2-(2-(2-(2-(4-(2-phenoxyacetyl)piperazin-1-yl)acetamido)ethoxy)ethoxy)ethyl)pentanamide (Probe 3). 


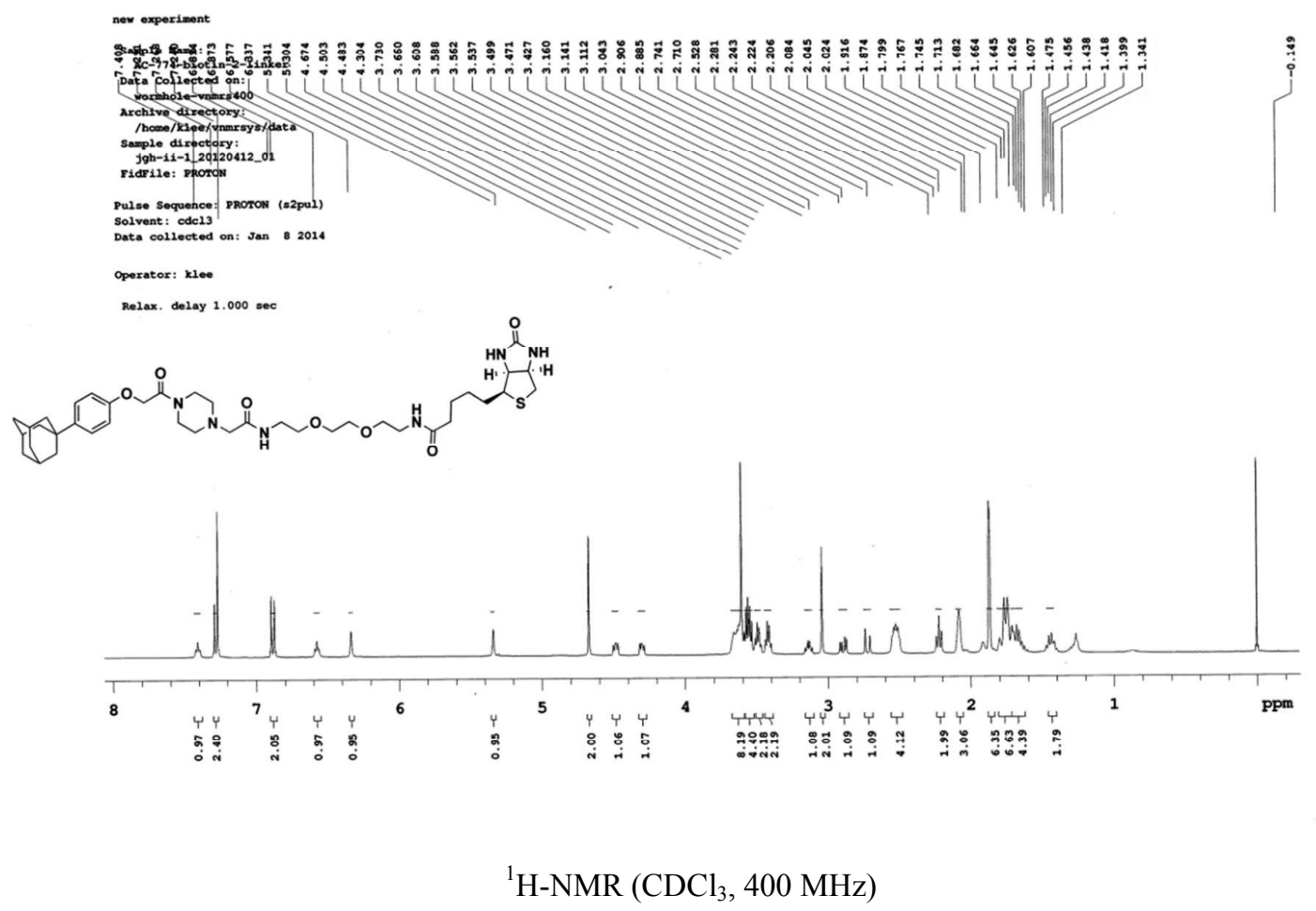

Figure S17. ${ }^{1} \mathrm{H}-\mathrm{NMR}$ spectrum of $N$-(2-(2-(2-(2-(4-(2-(4-adamantan-1-yl-phenoxy)acetyl)piperazin-1yl)acetamido)ethoxy)ethoxy)ethyl)-5-((3aS,4S,6aR)-2-oxohexahydro-1H-thieno[3,4-d]imidazol-4yl)pentanamide (Probe 4). 


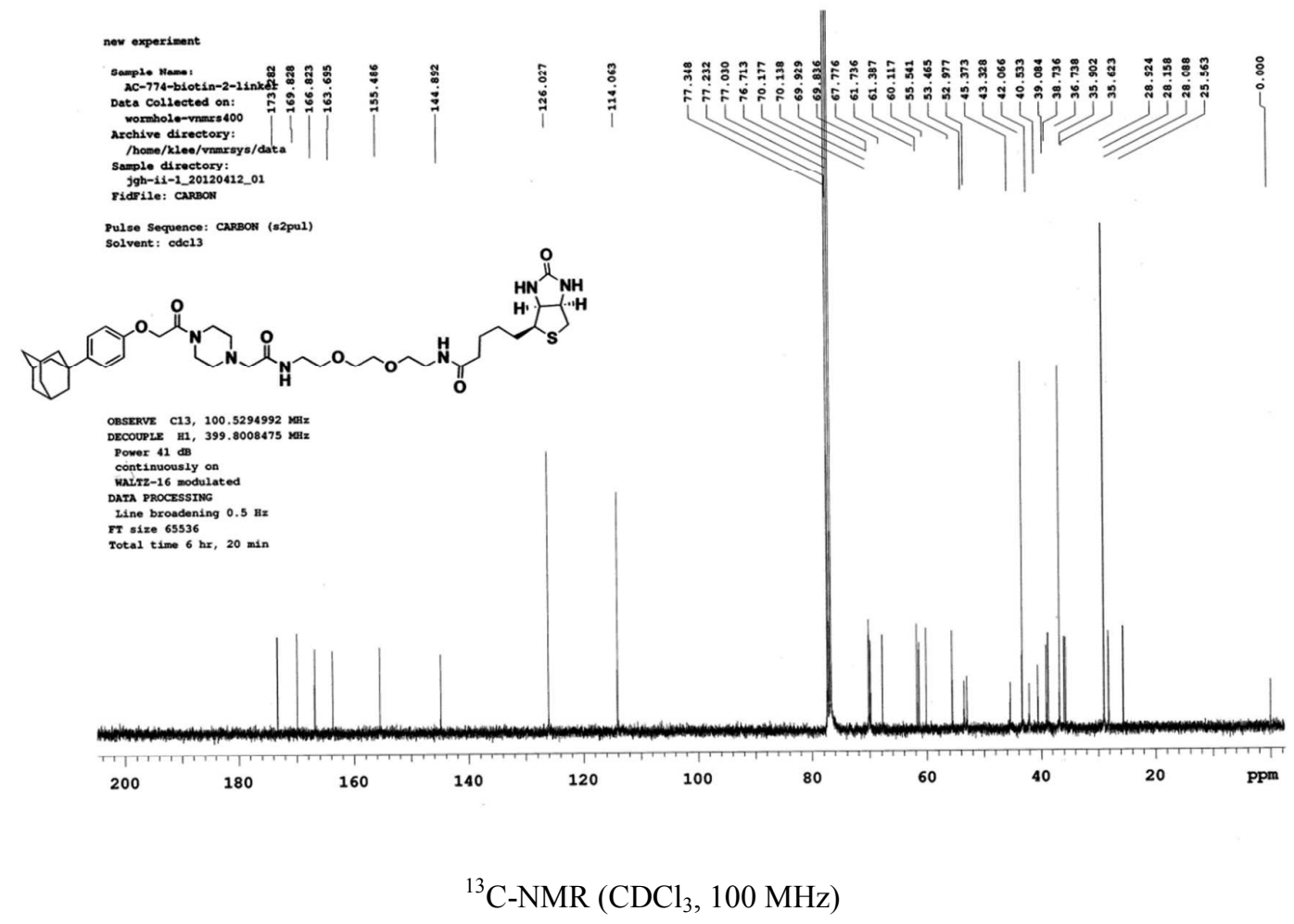

Figure S18. ${ }^{13} \mathrm{C}-\mathrm{NMR}$ spectrum of $N$-(2-(2-(2-(2-(4-(2-(4-adamantan-1-yl-phenoxy)acetyl)piperazin-1yl)acetamido)ethoxy)ethoxy)ethyl)-5-((3aS,4S,6aR)-2-oxohexahydro-1H-thieno[3,4-d]imidazol-4yl)pentanamide (Probe 4). 


\section{Abbreviations}

EPO, erythropoietin; HIF, hypoxia-inducible factor; HRE, hypoxia-response element; HSP, heat shock

protein; NBD, nucleotide-binding domain; OCR, oxygen consumption rate; SBD, substrate-binding domain; VEGF, vascular endothelial growth factor

\section{References}

(1) Lee, K., Lee, J. H., Boovanahalli, S. K., Jin, Y., Lee, M., Jin, X., Kim, J. H., Hong, Y. S., and Lee, J. J. (2007) (Aryloxyacetylamino)benzoic acid analogues: A new class of hypoxia-inducible factor-1 inhibitors. J. Med. Chem. 50, 1675-84.

(2) Naik, R., Won, M., Ban, H. S., Bhattarai, D., Xu, X., Eo, Y., Hong, Y. S., Singh, S., Choi, Y., Ahn, H.-C., and Lee, K. (2014) Synthesis and structure-activity relationship study of chemical probes as hypoxia induced factor- $1 \alpha /$ malate dehydrogenase 2 inhibitors. J. Med. Chem. 57, 9522-38.

(3) Cassel, J. A., McDonnell, M. E., Ilyin, S., and Reitz, A. B. (2012) Novel inhibitors of heat shock protein Hsp70-mediated luciferase refolding that bind to DnaJ. Bioorg. Med. Chem. 2012, 20, 3609-14. 